\title{
Teaching of Image Processing of Macroscopic, Microscopic, Knowledge Systems and Applications
}

\author{
Weilan Wang \\ School of Math and Computer Science, Northwest University for Nationalities, Lanzhou, China \\ wangweilan@xbmu.edu.cn
}

\begin{abstract}
Keywords: Digital Image Processing Teaching; Macroscopic Grasp; Microscopic Understanding; Knowledge Systems
\end{abstract}

\begin{abstract}
How to do well in the teaching of digital image processing for graduate students? A teaching philosophy were shown on digital image processing that is the macroscopic grasp and microscopic understanding, knowledge structure system and practical problems research in the applications. From the basic knowledge to practice, cultivate students' ability about to solve practical problems, to do experiment, to design software, to discover and analyze problems, to summarize and writing ability in Chinese and English etc. Many years of teaching practice has proved the effectiveness of our methods.
\end{abstract}

\section{Introduction}

Image processing is methods and techniques of denoising, enhancement, restoration, segmentation, feature extraction and encoding processing the images by computer, the goals of processing related to applications, mainly to meet people's visual and other technical requirements. Image processing is an application of signal processing in image domain. At present, most images are stored in digital form, so image processing is often referred to as digital image processing. In addition, processing method based on optical theory still occupy an important position. Digital image processing is a subclass of signal processing, which is closely related to computer science, artificial intelligence, image analysis and understanding, computer vision, pattern recognition and so on. Moreover, images are the most natural a natural language, the image processing can draw on the method of natural language processing. From the three levels of image processing, image analysis and image understanding, digital image processing is an indispensable foundation. In the teaching of digital image processing, we should not only see the forest, but also hold the trees, and then go deep into the branches, trunk and leaves of the trees. Digital image processing is also a comprehensive subject, with basic content and technical characteristics different from other courses. This paper attempts to summarize the teaching of digital image processing from the perspectives of macro, micro, knowledge structure and application.

\section{Macroscopic Hold of Digital Image Processing}

Research Contents of Image Processing. Human cognition and understanding of the environment, more than $70 \%$ will be through the visual system to obtain. As a subject, digital image processing was formed in the early 1960s. The purpose of early image processing is to improve the quality of images, so as to achieve a certain visual effect. The rapid development of digital image processing, mainly from the development of computers and mathematics, especially the establishment and improvement of discrete mathematics theory, as well as the rapid growth in the application of agriculture, animal husbandry, forestry, environment, military, industry and medicine. These applications involve image acquisition, storage, transmission, transformation, enhancement, restoration, compression, segmentation, feature extraction, classification and recognition and so on, thus forming the basic content of digital image processing research.

Three Levels of Image Technology. Image technology can be divided into three related and different levels according to their characteristics: image processing, image analysis and image understanding. 
Image processing: the input is image, and the output is still the image. This level is pixel level operation, so the amount of data is large and the degree of abstraction is low.

Image analysis: the input is image, the output is data. This level is the operation of the target level, the data size decreases and the degree of abstraction increases. Image analysis is to detect and measure the object of interest in the image, in order to obtain its objective information, so as to establish the description of the image, whose input is the image, and then output the data through image processing. The fields include content-based image retrieval, face recognition, facial expression recognition, optical character recognition, handwriting recognition, medical image analysis, video object extraction, and so on.

Image understanding: the input is images and the output is the explanation. The level is symbolic operation, relative to the smallest amount of data, the highest degree of abstraction, and the highest degree of semantics. Image understanding takes the image as the object and the knowledge as the core. It studies the relation between the objects and the objects in the image, what scene the image is and how to apply the scene, that is, the semantic understanding of the image. Image understanding belongs to senior operations in image engineering, which focuses on the properties of the target's relationship based on image analysis, and gets the understanding of the meaning and the interpretation of the original objective scene in the image, and further guide and plan the behavior of the machine. The object of the image understanding process is the symbol abstracted from the description, and there are many similarities between the process and the method with the human thinking reasoning.

Image engineering is an organic combination of the three, and its hierarchical relationship is shown in Figure 1. Image processing, image analysis and image understanding have their research contents and different technical means. The dependence between the three fully illustrates the basic role of image processing.

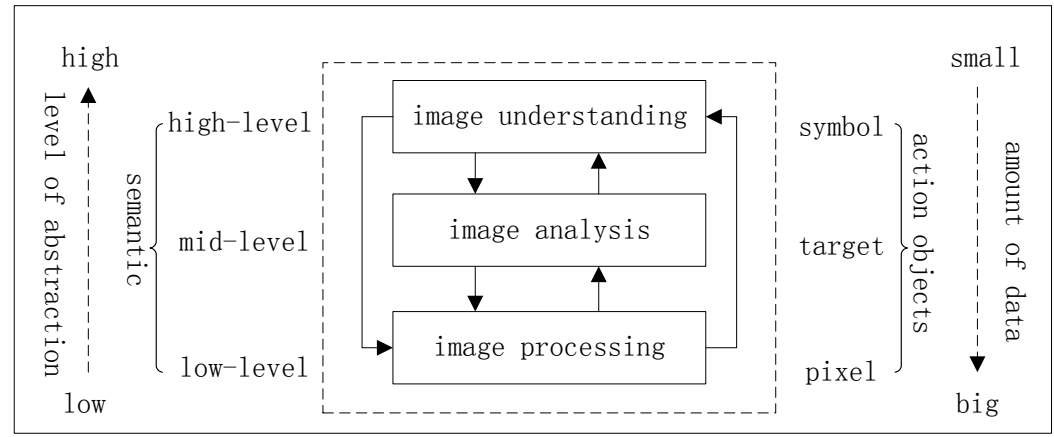

Figure 1. Hierarchical relation of image engineering

The Role of Image Segmentation and Knowledge Base in Image Engineering. After image processing, a key step of further segmentation as the image analysis and understanding, the results will directly affect the target feature extraction and description, and object recognition, classification and interpretation. In the process of image low-level processing, middle-level processing and high-level processing, the support function of the knowledge base makes the processing of each level more effective. The construction of the knowledge base and its specific content are related to the practical application. As shown in figure 2, the "preprocessing" of the low-level processing is just the method of image processing, such as image enhancement, restoration, and so on; the "representation and description" of middle-level processing corresponding to image analysis; the "recognition and interpretation" of high-level processing corresponds to image understanding. 


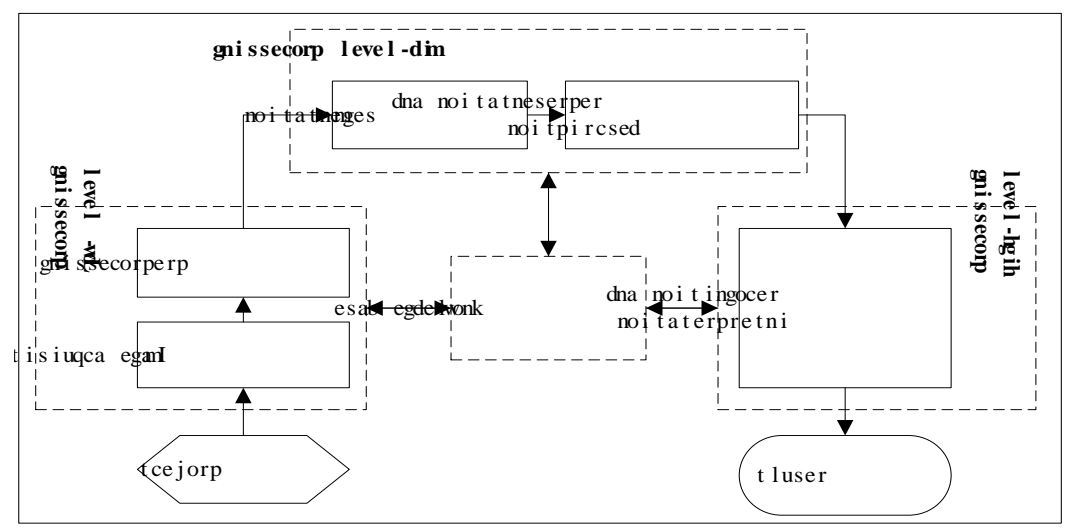

Figure 2. The role of image segmentation and knowledge base in image engineering

Image Processing and Related Disciplines. One of the theoretic foundation of digital image processing is mathematics, which involves matrix operation, linear and nonlinear transformation, orthogonal transformation, signal processing and so on, matrix operations, linear and nonlinear transformation, orthogonal transformation, signal processing. In addition, image processing is closely related to image analysis, image understanding, pattern recognition, computer vision, graphics and so on. If there is no understanding of the image, it is impossible to discuss the detection, classification and recognition of image targets. From the image to the symbol pattern recognition, so that the image understanding, or from the image to the data and then to achieve image understanding, graphics, etc., new concepts, tools and theories emerged continuously. It is irrelevant whether the image understanding model is consistent with the human cognitive process, and the key is that the method is effective, which can make the research, development and application of image processing more and more widely. As shown in Figure 3, the relationship between image processing and related disciplines[1]. Of course, images are the most natural a natural language, so the method of natural language processing should be referred and introduced to the image understanding.

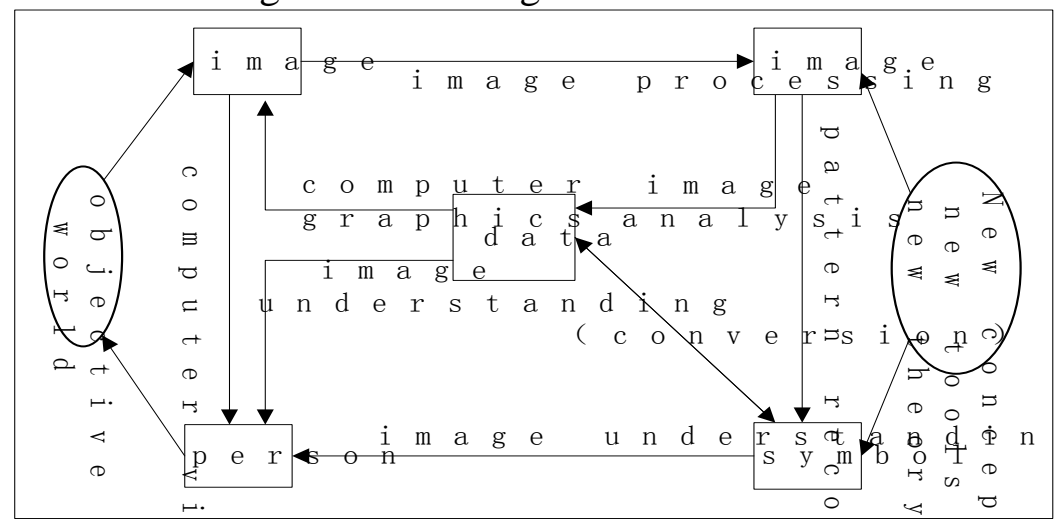

Figure 3. Image processing and related disciplines

Image processing takes input images or transforms them or processes them into another desired image, which transforms, or processes the input images into another desired image. The conversion or handling, for example, image enhancement processing, highlight the details of the image in order to meet further operational requirement, so that the output image has a higher signal-to-noise ratio. In the research of computer vision, image processing technology is used to preprocess and extract feature. Pattern recognition uses the statistical features or structural information extracted from the image to determine the known categories of the image. Such as text recognition, fingerprint identification, face recognition and so on. In computer vision, pattern recognition technology is usually used to segment, identify and classify some parts of an image.

To the objective world, the research contents and objectives of many subjects are related to computer vision, including image processing, pattern recognition or image recognition, scene analysis, image understanding, and so on. As far as computer vision is concerned, besides image processing and pattern 
recognition, spatial shape description, geometric modeling and process of cognition are also included. Computer vision studies how to make machines "look" like people, and make "responses", such as understanding the scene and reflecting it in a timely manner. Image understanding is the ultimate goal of computer vision. In the establishment of computer vision system, it is also necessary to use the related techniques in the subject described in Figure 3, but the content and application fields of computer vision research are more extensive than those of other disciplines.

\section{Microscopic Understanding of Digital Image Processing}

Digital Image. There are two types of vector and bit mapping images about digital image storage and display. A vector diagram is defined mathematically as a series of points connected by lines, which are object oriented images or drawing images. The graphic elements in vector file as the object, each object is a self-contained entity, it has the color, shape, contour, size and screen position and other attributes. The greatest advantage of vector graphics is that they are undistorted and also independent of resolution, whether magnified, reduced, or rotated; The biggest drawback is that it is difficult to display realistic images with rich color levels. A bit map, that is, a raster image, consists of pixel points, and the storage format is called a bitmap image. The stored data is the number of pixels describing the image. The image formats include two binary images, color images, pseudo color images and three-dimensional images. Its display formats include BMP, GIF, JPEG, JPEG2000, tiff, PSD, PNG, SWF, SVG, PCX, DXF, WMF, EMF, LIC, EPS, TGA, and so on. A description of the stored data structure is used to further understanding and analysis images.

The elementary unit of image is pixel, the distance between adjacent pixels ranks reflects the spatial resolution. Image sampling and quantization is a manifestation of the spatial resolution and gray scale resolution, and the level of spatial resolution size and grayscale resolution is important indicator of image quality. The adjacency, adjacency, connectivity, distance measurement, boundary representation, and region description among pixels are tools for further understanding the inner of region, between regions and boundary of image. Commonly used color space classification, mainly for hardware oriented color model (RBG, CMY, YCrCb) and visual perception of color models (HSI, HSV, HSB, Lab). The conversion between image formats can be convenient for different applications and research.

Characteristics of Image Processing. Image processing is an application of signal processing in two-dimensional signal that is image domain. Image processing, namely, processing and analysis to meet people's visual and psychological requirements, as well as other application requirements of the technology. The image is stored in digital form, so digital image processing has the following characteristics: Large amount of information, requiring faster processing; the correlation between pixels is strong and the compression potential great; image processing, including spatial-domain and frequency-domain methods, is two methods of recognizing and processing digital images from different angles, and Fourier and other orthogonal transformations play a crucial role in image processing; the image processing technology is comprehensive, and a high requirement for mathematics.

\section{Knowledge System for Digital Image Processing}

Fundamentals of Image Processing. The foundations of mathematics include mathematical analysis, linear algebra, statistics, stochastic processes, differential equations, functional analysis, etc., as well as signal processing systems and so on.

Physiological Basis of Image Processing. Human visual perception still plays an important role in image processing technology. Even people often say that without human vision, there is no image, no image processing. Of course, vision, cognition, psychology, optics and color science are complicated problems with images. They are all involved in digital image processing, and more knowledge need further study to master by the students themselves.

Basic Methods of Image Processing. To improve the visual quality of images so as to achieve the pleasing purpose, some features or special information contained in the image are extracted, in order to computer analysis, transform, encode and compress the image data, so that the storage and transmission 
of the image, which is the purpose of image processing. Therefore, the rich content of image enhancement, restoration, image transformation, compression coding, segmentation, edge representation, feature extraction and representation are the basic methods.

The Development of Image Processing Technology. Image processing and analysis is an exciting emerging field [2], from an image input, processing to output, the processed image is generally observed and evaluated by human vision, so it is greatly influenced by human factors. Because the human visual system is very complex, it is greatly affected by environment conditions, visual properties, people's emotional preferences and knowledge status. As an aspect of image quality evaluation, further research is needed. Image processing is a fast developing subject, which will develop in the direction of high speed, high resolution, stereoscopic, multimedia, intelligence and standardization. Meanwhile the combination of images and graphics moves toward three-dimensional imaging or multidimensional imaging. Furthermore combined with multimedia technology, more and more hardware chips, many of the functions of image processing will be solidified in the chip will have more extensive application areas. At last in the field of image processing, new theories will be introduced and some new algorithms are proposed.

Digital Image Processing Tools. Digital image processing is an applied and practical subject. Besides the mathematical, the visual system and the signal processing foundation, it is necessary to master the programming tools. From input digital image to output processed image, this process includes the application of image processing platform and the realization of image processing algorithm. The commonly used image processing programming tools are: MATLAB, Open $\mathrm{CV}, \mathrm{C}, \mathrm{C}++, \mathrm{VC}$, etc.

\section{The Teaching of Digital Image Processing}

The Application Direction of Image Processing and the Limitations of Related Methods. Digital image processing has a wide range of applications, involving all aspects of national security, economic and social development and human life and work. It can be summarized to 10 aspects[3]: aeronautics and astronautics, biomedical engineering, communication engineering, industry and engineering, military and public security, robot vision, video and multimedia systems, scientific visualization, electronic commerce, and digital protection of culture, art and cultural heritage, etc.

Digital image processing is an applied discipline and an engineering discipline. In the teaching, the application is the starting point and the end-result, in particular, the contents and methods of processing are introduced from the actual image. Then explore and solve the problem, and discuss the actual effect. At the same time to illustrate the limitations of various image processing methods, or there is no universal image processing method to solve all problems.

Solve Problems is Always the Driving Force of Study and Exploration. In our teaching process, the actual problems are the digital protection of Thangka and Tibetan character recognition. For example, the pretreatment of Thangka images, segmentation algorithms and restoration algorithms for different damage regions are studied. On the one hand, the general methods of image processing are validated in complex images with no obvious foreground and background for Thangka image, and on the other hand, because of the particularity of Thangka image, some methods do not work well or adapt to the Thangka image. Through the improvement of the algorithm or the design of the model, it can provide better technical support for Thangka image in enhancement, segmentation of damaged region, virtual restoration and related applications. In order to study and solve practical problems, students must read the latest literature both at home and abroad, so that students can compare existing approaches to frontier technologies. Improve students' reading ability, practical ability and writing ability.

Learning in Order to Practice. Over the years, our students have carried out fruitful learning, application and practice, in the field of Thangka digitization, restoration of damaged regions of Thangka images, feature extraction and image retrieval and so on. The following examples illustrate the application of some students.

In the restoration of the damaged area of Thangka image: in order to improve the speed of exemplar-based image inpaining algorithm, an improved method is proposed to repair the damaged region by using the information around the damaged patchs[4]. For the deficiencies of exemplar-based 
broken Thangka image inpainting, a new algorithm are proposed by improvement of computing method of confidence and the improvement of computing method of isophote intensity, and the problem that optimum exemplar patch is not exclusive has been solved. The experimental results show that the improved algorithm can not only get a satisfied inpainting result but also improve repairing efficiency[5]. A fast image inpainting algorithm based on TV model, based on the analysis of local features, according to the boundary pixel priority of damage region, first filter stratification and the damaged area, and then to the priority level of the circulation from the outside to the inside of the repair, achieved good restoration effect[6]. When the missing region is unique texture patch, the unreasonable texture is filled in missing region because similar exemplar of damaged patch could not be found, a new image inpaining method based on eight directions symmetrical exemplar is proposed, which is suitable for damaged image containing local symmetry[7].

In the segmentation of complex damaged image: the damage region should be segmented before inpainting, and how to automatically segment the damaged region is also worth discussing. A segmentation method of spotted type damaged Thangka images based on gray morphology and maximum entropy threshold is proposed, and a practical segmentation method of damage region is realized[8]. In addition, a segmentation method of the damage region of Thangka images combined with color and texture features has also achieved good results[9].

In the feature extraction and retrieval of image: the Buddhist images of Thangka, the characteristics are extracted and represented of headdress and gesture which are to distinguish the different gods[10]; research on Thangka image retrieval based on the feature of gods gesture[11]. These lay a foundation for semantic-based Thangka image retrieval. Extracting the features of Thangka images from the global and local, and then some methods are obtained, such as how to distinguish Thangka and non-Thangka image[12], improved local accumulate histogram-based Thangka image retrieval ${ }^{[13]}$, headdress detection of Thangka portrait image based on saliency map[14], and Thangka image classification based on sub-block color histogram[15] ,etc. Moreover, Thangka image of the gods with the categories of silence and wrathful represent different religious meanings, and how to detect the head light and backlight of the silence classes, and the flame of wrathful classes, it is also the research content of feature extraction and image classification, and obtains better detection results[16,17]. Based on the related knowledge and rules reasoning of Buddhist Thangka, some knowledge base system of Thangka image are constructed[18,19].

\section{Summary}

Graduate course teaching of digital image processing and its application, should grasp the basic position of digital image processing in related disciplines and broad application prospects from the macroscopic; should master the characteristics of digital image, the method of pixel level processing, the format of image storage and the tools of image processing etc., from the microscopic. On this basis, the actual problems will be studied and discussed, to improve students' ability to solve problems.

\section{Conclusions}

Digital image processing is a subject closely related to theory and practice. The way of teaching and learning is worthy of constant study, exploration and practice. The training of talents in this course and its fields should focus on basic knowledge, practical ability and professionalism. We should combine the basic knowledge with the application, and combine the depth and breadth of the subject. Around the project, understand the research situation at home and abroad and deep-going study and practice. At the same time, we should have a comprehensive understanding of the main methods and developments in related fields, such as image analysis and understanding, computer vision, pattern recognition and other related disciplines. In addition, the practice ability is reflected in the flexible use of professional knowledge, the experimental ability to solve practical problems, and the ability to find and analyze problems in experiments, and summarizing and writing ability in Chinese and English etc.. It is also important for instructors to develop interest in their students and to be strict in their work. 


\section{Acknowledgements}

This work was supported by the demonstrative degree program of Northwest University for Nationalities (No.1671280207), and the personnel training program of State Ethnic Affairs Commission.

\section{References}

[1] ZHANG Yujin. Fundamentals of image processing and analysis, Higher Education Press, 2002.

[2] Tony F.Chan, Jianhong Shen. Image Processing and Analysis---Variational, PDE, Wavelet and Stochastic Methods. Science Press, 2009.

[3] Rafael C.Gonzalez, Richard E.Woods.Digital Image Processing.Publitioning House of Electronics Industry.2006.

[4] J. van der Geer, J.A.J. Hanraads, R.A. Lupton, The art of writing a scientific article, J. Sci. Commun. 163 (2000) 51-59.

[4] Huaming Liu, Weilan Wang and Hui Xie, Thangka Image Inpainting Using Adjacent Information of Broken Area, International MultiConference of Engineers and Computer Scientists Hong Kong, 19-21 March. (2008) 646-649.

[5] LU Xiaobao, WANG Weilan, Improved exemplar-based inpainting algorithm for broken Thangka images, Journal of Computer App lications,30(4),(2010)943-946\&952.

[6] Huaming Liu, Weilan Wang and Hui Xie, Thangka Image Inpainting Using Adjacent Information of Broken Area, International MultiConference of Engineers and Computer Scientists,Hong Kong, 19-21 March, (2008) 646-649.

[7] Xiaobao Lu, Weilan Wang, Duojie Zhuoma, A Fast Image Inpainting Algorithm Based on TV Model, Proceeding of the International MultiConference of Engineers and Computer Scientists Vol II.IMECS, Hong Kong, March 17-19(2010)1457-1460

[8] Yanjun Jia,Weilan Wang,Tiejun Wang,Baojuan Luo,A Novel Image Inpainting Method Based on Eight-Direction Symmetrical Exemplars,6rd International Congress on Image and Signal Processing, ( 2013)303-307

[8] Baojuan Luo, Weilan Wang, Yanjun Jia,Wanpin Gao, A Segmentation Method for Spotted- partten Damaged Thangka Image Combining Grayscale Morphology with Maximum Entropy Threshold, 6rd International Congress on Image and Signal Processing,(2013)537-542

[9] Baojuan Luo, Weilan Wang, Wenjin Hu, Wenbin Lv, Damaged Regions Segmentation on Thangka Image Combining Color and Texture Features, International Journal of Digital Content Technology and its Applications, 10(5) (2016 )131-143

[10] Qian Jianjun, Wang Weilan. Main Feature Extraction and Expression for Religious Portrait Thangka Image. The 9th International Conference for Young Computer Scientists. (2008). 803-807.

[11] Jianjun Qian, Weilan Wang, Religious Portrait Thangka Image Retrieval Based on Gesture Feature, Chinese Conference on Pattern Recognition, (2009)568-572.

[12] Lu Yin, Weilan Wang, Danchun Yang, Study on How to Distinguish Thangka and Non-Thangka Image,Proceeding of the International MultiConference of Engineers and Computer Scientists Vol II , Hong Kong, March 17-19(2010)1476-1480.

[13] Xiaojie Li, Weilan Wang, Wei Yang, Improved Local Accumulate Histogram-based Thangka Image Retrieval, International Conference on Image Analysis and Signal Processing, Xiamen, Fujian, China. April 12-14(2010)318-321.

[14] Lu Yin, Weilan Wang, Headdress Detection Based on Saliency Map for Thangka Portrait Image, Proceedings of 12th IAPR Conference on Machine Vision Applications, Nara, Japan (2011) 487-490.

[15] Wanpin Gao, Weilan Wang, Yanjun Jia and Baojuan Luo, Thangka Image Classification Based on Sub-Block Color Histograms. International Symposium on Signal Processing, Biomedical Engineering, and Informatics,(2013)1102-1109 
[16] Wentao Shen, Weilan Wang, The detection of Buddha's circle head light and backlight in Thangka Image, Conference on Computer Science and Artificial Intelligence (2016)517-527.

[17] Yuan Chen, Cheng Peng, Wei Lan Wang, Image Detection of the Wrathful Classes Thangka Based on Color and Shape Feature, International Conference on Network and Information Systems for Computers,(2015)514-518

[18] Wei Yang, Xiaojie Li,Weilan Wang, Structure of Semantic Knowledge Database Rules-Based Religious Portrait Thangka Image, International Conference on Networking and Digital Society Volume 2, Wenzhou,China,30-31 May (2010)59-62

[19] Wang Tiejun, Wang Weilan, A Novel Modified Evolutionary Algorithm based Image Retrieval Framework: Theoretical Analysis and Applications. International Journal of Signal Processing, Image Processing, 9(1) (2015)221-230

[20] Wang Tiejun, Wang Weilan, Research on New Multi-Feature Large-Scale Image Retrieval Algorithm based on Semantic Parsing and Modified Kernel Clustering Method, International Journal of Security and Its Applications, 10(1)(2016)139-154. 